\title{
A Possibility Priority Degree Analyzing Process for Multiple Attributes Decision Making Problems
}

\author{
Mingming Hu, Xinmiao Ye, Jibin Lan ", Fang Liu \\ College of Mathematics and Information Science, Guangxi University, Nanning, China \\ Email address: \\ lanjibin@gxu.edu.cn (Jibin Lan),hmm0112@126.com (Mingming Hu) \\ ${ }^{*}$ Corresponding author
}

To cite this article:

Mingming Hu, Xinmiao Ye, Jibin Lan, Fang Liu. A Possibility Priority Degree Analyzing Process for Multiple Attributes Decision Making Problems. European Business \& Management. Vol. 4, No. 2, 2018, pp. 44-54. doi: 10.11648/j.ebm.20180402.11

Received: December 6, 2017; Accepted: January 4, 2018; Published: January 19, 2018

\begin{abstract}
A multiple attributes decision making model is wildly used and studied. The goal of multiple attributes decision making problems is to select a perfect alternative. The existed methods pay attention to rank the alternatives and suggest a best alternative to decision makers. However, there is risk hiding on the priority order. When accepting the order, decision makers undertake the risk at the same time. It is unknown for decision makers. To show the advantages and disadvantages for each alternative, and the risk of a selection, we propose a possibility priority degree analyzing model. With this model, decision makers can be aware of the possibility of priority degree, similar degree and the priority risk, and then make decision. It will effectively reduce the decision risk and improve the decision efficiency.
\end{abstract}

Keywords: MADM, Possibility, Priority Degree, Alternatives, Attributes

\section{Introduction}

A multiple attribute decision making (MADM) [1] problem includes several alternatives. Each alternative can be described by an attribute system. The goal of solving MADM problem is to select a perfect one from alternatives. Since 1950s, it has been wildly used (Job selection [2]; Product design [3]; leisure time allocation [4]; making business investment decision [5]; Selecting military hardware [6]) and studied (Dominance method [7]; Satisficing method [8]; Maximin method [1]; Maximax method [1]; Lexicography method [1]; Additive weighting method [9]; Non-metric scaling method [10]). The dominance method shows that if some one alternative has higher attribute values for all attribute, we say that this alternative "dominates" the others [1]. The satisfying method shows that the decision maker supplies the minimal attribute values he can accept for each of the attributes. The alternatives whose attribute values are better than the minimal acceptable goal can be taken as feasible alternatives [1]. The maximin method is to note the lowest value of each alternative and select the alternative with the most acceptable value of its lowest attribute [1]. The maximax methods are to identify the highest attribute value of each alternative and select the alternative to the largest value [1]. Lexicography method is to consider the most important attribute to decision maker and select the alternative to the most important attribute value [1]. The additive weighting method is to weight each attribute value by a measure to get a weighted average of the contribution to each alternative and select the alternative to the highest weighted average [1]. The non-metric scaling method is to specify an ideal object (the most preferred values on each of the attributes) and determine the distance between each of the other alternatives and this ideal object. The alternative which is closest to the ideal object would be the chosen alternative [10].

These methods for MADM problems can be classified into three kinds. The first one is the dominance method. The decision is accurate. And the best choice is determined. It can't change into anyone in anyplace and anytime. But it is not practical. The second one is the satisfying method, Maximin method, Maximax method, lexicography method. These methods pay attentions to single attribute, and make decision largely depending on single attribute value. In this way, the untaken attribute value missed in the process of decision making. The third one is the additive weighting method and non-metric method. These two methods integrate all the attribute information. It is an average method. However, this 
kind of method neglects the worse attribute value and best attribute value. Thus, except for the first kind (one alternative has advantage for all attributes), the other two kinds of method comparing alternatives, generating that one alternative is better than another in $100 \%$ percentage and providing a best alternative for decision maker are not sensible. Each alternative has both advantages and disadvantages. Alternatives can't be ordered only by their advantage $/$ disadvantage $/ w e i g h t e d$ average. It would miss the information of other aspects. Take the advantages for example, when you select a basketball player, only takes the advantage of player into account, a man with very well skills and very poor cooperation may be selected. However, in playing basketball, cooperation is very important.

How to do multiple attributes decision making problem? A priority-possibility degree analyzing (PPDA) method will be proposed in this paper. The paper is organized as follows: Section 2 will introduce the MADM problem and existed MADM method. The priority-possibility degree analyzing method will be introduced in Section 3. In Section 4, the proposed analyzing method will be extended and the attribute weight will be considered. This paper will be concluded in Section 5.

\section{The Existed MADM Method}

A MADM problem is to select alternatives from a group of alternatives. Suppose there are $m$ alternatives: $x_{1}, x_{2}, \ldots, x_{m} ;$ an quantitative attribute system $\left(u_{1}, u_{2}, \ldots, u_{n}\right)$, in which each pair of attributes is independent, can be taken to express the characteristic of each alternatives. And all the attribute values $\left(a_{i j}\right)$ are known uniquely. The MADM problem can be shown in Table 1 .

Table 1. A MADM problem.

\begin{tabular}{lllll}
\hline & $\mathbf{u}_{\mathbf{1}}$ & $\mathbf{u}_{2}$ & $\ldots$ & $\mathbf{u}_{\mathbf{n}}$ \\
\hline $\mathrm{x}_{1}$ & $\mathrm{a}_{11}$ & $\mathrm{a}_{12}$ & $\ldots$ & $\mathrm{a}_{1 \mathrm{n}}$ \\
$\mathrm{x}_{2}$ & $\mathrm{a}_{21}$ & $\mathrm{a}_{22}$ & $\ldots$ & $\mathrm{a}_{2 \mathrm{n}}$ \\
$\ldots$ & & & $\ldots$ & $\ldots$ \\
$\mathrm{x}_{\mathrm{m}}$ & $\mathrm{a}_{\mathrm{m} 1}$ & $\mathrm{a}_{\mathrm{m} 2}$ & $\ldots$ & $\mathrm{a}_{\mathrm{mn}}$ \\
\hline
\end{tabular}

According to the numerical multiple attribute decision information $\left(A=\left(a_{i j}\right)_{m \times n}\right)$, which one should we choose?

There are several methods for this problem.

To make the numerical information of different attribute comparable, normalize [11-13] the attribute value $A=\left(a_{i j}\right)_{m \times n}$ to $B=\left(b_{i j}\right)_{m \times n}$ by

(i) If the $j^{\text {th }}(1 \leq j \leq n)$ attribute is benefit attribute, then

$$
b_{i j}=\frac{a_{i j}}{\max _{(1 \leq i \leq m)} a_{i j}}, 1 \leq i \leq m .
$$

(ii) If the $j^{\text {th }}(1 \leq j \leq n)$ attribute is cost attribute, then

$$
b_{i j}=\frac{\min _{(1 \leq i \leq m)} a_{i j}}{a_{i j}}, 1 \leq i \leq m .
$$

After that, the style of all attributes change to benefit. And the value of all attributes comparable.

a. Dominance method [7]
Denote one alternative by $x_{i} \sim\left(b_{i 1}, b_{i 2}, \ldots, b_{i n}\right)$ and another by $x_{k} \sim\left(b_{k 1}, b_{k 2}, \ldots, b_{k n}\right)$. Then we say that the second alternative dominates the first $x_{k} \succ x_{i}$ if $b_{k j} \geq b_{i j}$ for all $j$, and further $b_{k j^{\prime}}>b_{i j^{\prime}}$ for some $j^{\prime}$.

b. Satisficing method [8]

The decision maker supplies the minimal attribute values he will accept for each of the attribute $\left(b_{1}^{0}, b_{2}^{0}, \ldots, b_{n}^{0}\right)$, the alternative $x_{i}$ is taken as a feasible alternative if $b_{i j} \geq b_{j}^{0}$ for all $j$. After this process, we are still left with a number of feasible alternatives.

c. Maximin method [1]

This method takes the mean of an old saying that"the chain is only as strong as its weakest link". Select the weakest attribute value $b_{i}^{0}$ of $x_{i}$ by

$$
b_{i}^{0}=\min \left\{b_{i 1}, b_{i 2}, \ldots, b_{i n}\right\}, i=1,2, \ldots m .
$$

Then $x_{i^{*}}$ will be selected out as the best alternative if

$$
b_{i^{*}}^{0}=\max _{(1 \leq i \leq m)} b_{i}^{0} .
$$

d. Maximax method [1]

Select the strongest attribute value $b_{i}^{*}$ of $x_{i}$ by

$$
b_{i}^{*}=\max \left\{b_{i 1}, b_{i 2}, \ldots, b_{i n}\right\}, i=1,2, \ldots m .
$$

$x_{i *}$ will be selected out as the best alternative if

$$
b_{i^{*}}^{*}=\max _{(1 \leq i \leq m)} b_{i}^{*} .
$$

e. Lexicography method [1]

Suppose the attributes are ordered so that $u_{1}$ is the most important attribute to the decision maker, $u_{2}$ is the next most important, and so forth. Then take

$$
\aleph^{1}=\left\{x_{i^{*}} \mid b_{i^{*} 1}=\max _{(1 \leq i \leq m)} b_{i 1}, 1 \leq i^{*} \leq m\right\} .
$$

If $\aleph^{1}$ has a single element, then this one is the most preferred alternative. Else, consider

$$
\aleph^{2}=\left\{x_{i^{*}} \mid b_{i^{*} 2}=\max _{\left(i \in\left\{k \mid x_{k} \in \aleph^{1}\right\}\right)} b_{i 2}, i^{*} \in\left\{k \mid x_{k} \in \aleph^{1}\right\}\right\}
$$

If $\aleph^{2}$ has a single element, then this one is the most preferred alternative. Else, continue this process until either (i) some $\mathfrak{K}^{*}$ with only a single element is found, which is the most preferred alternative or (ii) all attributes have been considered, in which case if the remaining set contains more than one maximal element, they are considered to be equivalent [1].

f. Additive weighting method [9]

We can get the normalized weight $\left(w_{1}, w_{2}, \ldots, w_{n}\right)$ for each attribute by subjective weights and objective weights (The subjective methods are to determine weights solely according to the preference or judgments of decision makers. Then apply some mathematic methods such as the eigenvector method, weighted least square method, and mathematical programming models to calculate overall evaluation of each decision maker. The objective methods determines weights by solving mathematical models automatically without any 
consideration of the decision maker's preferences, for example, the entropy method, multiple objective programming, etc.)[14], where

$$
\sum_{j=1}^{n} w_{j}=1, w_{j} \geq 0, j=1,2, \ldots, n .
$$

Then, $x_{i *}$ will be selected out as the best alternative if

$$
\sum_{j=1}^{n} w_{j} \cdot b_{i^{*} j}=\max _{(1 \leq i \leq m)} \sum_{j=1}^{n} w_{j} \cdot b_{i j} .
$$

g. Non-metric scaling method [10]

Suppose $\left(w_{1}, w_{2}, \ldots, w_{n}\right)$ is the normalized attribute weights. The weighted attribute value $C=\left(c_{i j}\right)_{m \times n}$ can be gotten by

$$
c_{i j}=b_{i j} \cdot w_{j}, i=1,2, \ldots m ; j=1,2, \ldots, n .
$$

Denote one alternative by $x_{i} \sim\left(c_{i 1}, c_{i 2}, \ldots, c_{i n}\right)$ and another by $x_{k} \sim\left(c_{k 1}, c_{k 2}, \ldots, c_{k n}\right)$. The distance between any two points $x_{i}$ and $x_{j}$ is defined to be

$$
d\left(x_{i}, x_{k}\right)=\sqrt{\sum_{j=1}^{n}\left(c_{i j}-c_{k j}\right)^{2}} .
$$

Then, we locate an ideal object $x^{*} \sim\left(c_{1}^{*}, c_{2}^{*}, \ldots, c_{n}^{*}\right)$ in the alternative space, where

$$
c_{j}^{*}=\min _{(1 \leq i \leq m)} c_{i j}, j=1,2, \ldots, n .
$$

Thus, $x_{i^{*}}$ will be selected out as the best alternative if

$$
d\left(x_{i^{*}}, x^{*}\right)=\min _{(1 \leq i \leq m)} d\left(x_{i}, x^{*}\right) .
$$

Take an example from [1] to illustrate these methods.

Example 1 [1]. Suppose for a particular anticipated military requirement, say, within the general war mission, we must make a choice among designs for a future weapon system. Let us consider three possible types of system - call them X, Y, and $\mathrm{Z}$. The attributes (Range ( $\mathrm{n} \mathrm{mi}) \backslash$ Delivery time (hr) $\backslash$ Total yield (MT) \Accuracy (high-low) $\backslash$ Vulnerability (high-low) $\backslash$ Payload delivery flexibility (high-low)) are generated by careful political-military consideration of this particular

\begin{tabular}{|c|c|c|c|c|c|c|}
\hline & Range (n mi) & Delivery time (hr) & Total yield (MT) & Accuracy (high-low) & Vulnerability (high-low) & Payload delivery flexibility (high-low) \\
\hline $\mathrm{X}$ & 10,000 & 5 & 100 & Average & Average & High \\
\hline Y & 8,000 & 0.5 & 50 & Low & High & Low \\
\hline Z & 5,000 & 1 & 80 & High & Very low & Average \\
\hline
\end{tabular}
requirement within the overall mission and possibly also future uses of the proposed system. In this case, we can characterize each system uniquely by each set of attributes, which is shown in Table 2.

Table 2. A Weapon System Decision Problem.

The 1-9 scale [15] is taken for the corresponding qualitative ones in Table 2, which shows in Table 3.

Table 3. 1-9 numerical scale.

\begin{tabular}{llllll}
\hline Numerical Scale & $\mathbf{1}$ & $\mathbf{3}$ & $\mathbf{5}$ & $\mathbf{7}$ & $\mathbf{9}$ \\
\hline Vulnerability & Very high & High & Average & Low & Very low \\
Payload delivery flexibility/Accuracy & Very low & Low & Average & High & Very high \\
\hline
\end{tabular}

Then, the weapon system decision problem in Table 2 will change to Table 4.

Table 4. The Weapon System Decision Problem.

\begin{tabular}{lllllll}
\hline & Range (n mi) & Delivery time (hr) & Total yield (MT) & Accuracy & Vulnerability & Payload delivery flexibility \\
\hline X & 10,000 & 5 & 100 & 5 & 5 & 7 \\
$\mathrm{Y}$ & 8,000 & 0.5 & 50 & 3 & 3 & 3 \\
$\mathrm{Z}$ & 5,000 & 1 & 80 & 7 & 9 & 5 \\
\hline
\end{tabular}

To make the attributes comparable, normalize the information in Table 4, and we will get the decision information matrix.

Table 5. Comparable Numerical Values for the Problem.

\begin{tabular}{lllllll}
\hline & Range (n mi) & Delivery time (hr) & Total yield (MT) & Accuracy & Vulnerability & Payload delivery flexibility \\
\hline $\mathrm{X}$ & 1 & 1 & 1 & 0.7143 & 0.5556 \\
$\mathrm{Y}$ & 0.8 & 0.1 & 0.5 & 0.4286 & 0.3333 & 1 \\
$\mathrm{Z}$ & 0.5 & 0.2 & 0.8 & 1 & 1 & 0.4286 \\
\hline
\end{tabular}

The decision results of these existed methods are shown in Table 6.

Table 6. The decision results of existed method.

\begin{tabular}{lll}
\hline The existed method & Parameter & Results \\
\hline Dominance method & None & Invalid \\
\hline & $(0.5,0.1,0.5,0.4,0.3,0.4)$ & $\mathrm{X}, \mathrm{Y}, \mathrm{Z}$ \\
Satisficing method & $(0.8,0.1,0.5,0.4,0.3,0.4)$ & $\mathrm{X}, \mathrm{Y}$ \\
& $(0.5,0.1,0.8,0.8,0.3,0.4)$ & $\mathrm{Z}$ \\
\hline
\end{tabular}




\begin{tabular}{lll}
\hline The existed method & Parameter & Results \\
\hline Dominance method & None & Invalid \\
\hline & $(0.6,0.1,0.8,0.8,0.3,0.4)$ & Empty \\
Maximin method & None & $X>Z>Y$ \\
Maximax method & None & $X \sim Z>Y$ \\
Lexicography method & None & $X>Y>Z$ \\
Additive weighting method & $(0.05,0.1,0.1,0.4,0.15,0.2)$ & $X>Z>Y$ \\
Non-metric scaling method & $(0.04,0.1,0.1,0.4,0.16,0.2)$ & $\mathrm{Z}>\mathrm{X}>\mathrm{Y}$ \\
\hline
\end{tabular}

In this example, the Dominance method is unavailable. It can't be used to rank these alternatives. For the satisficing method, different decision maker provides different minimal attribute values, which will generate different ranking result of alternatives. Take four different groups of minimal attribute value in Table 6 for example. The minimal attribute values $(0.5,0.1,0.5,0.4,0.3,0.4)$ generate the result that all alternatives are feasible. If the minimal value of the first attribute changes to 0.8 , then alternative $\mathrm{Z}$ will be infeasible and alternatives $\mathrm{X}$ and $\mathrm{Y}$ are feasible. Besides, if the minimal value group changes to $(0.5,0.1,0.8,0.8,0.3,0.4)$, then only alternative $\mathrm{Z}$ is feasible. Furthermore, if the minimal value group changes to $(0.6,0.1,0.8,0.8,0.3,0.4)$, then none of the alternatives is feasible.

For the Maximin method, the alternative is only as strong as its weakest attribute. So the weakest attribute for alternative $X$ is Vulnerability, its value is 0.5556 . That for alternative $Y$ is Delivery time, its value is 0.1 . That for alternative $Z$ is also Delivery time, its value is 0.2 . Thus, the order of alternatives is $X>Z>Y$. It means that $X>Z, Z>Y$ and $X>Y$. In Table 5, comparing the each attribute value of alternatives, $X>Y$ is obviously right. However, both the other two relations cannot stand up. Let us pay attention to alternative $\mathrm{X}$ and $\mathrm{Z}$, in Table 5, if the attribute "Accuracy" or "Vulnerability" is very important in decision making, then the ranking may be reverse. In the same way, considering the alternative $Y$ and $Z$, you will generate the same result. Thus, this method would neglect the importance of attributes.

For the Maximax method, the alternative is as good as its best attribute. So the best attributes for alternative $X$ are Range $\backslash$ Delivery time $\backslash$ Total yield $\backslash$ Payload delivery flexibility, their value are all 1 . That for alternative $Y$ is Range, its value is 0.8 . That for alternative $Z$ are Accuracy/Vulnerability, their value are both 1 . Thus, the order of alternatives is $\mathrm{X} \sim \mathrm{Z}>\mathrm{Y}$. It means that $\mathrm{X} \sim \mathrm{Z}, \mathrm{Z}>\mathrm{Y}$ and $\mathrm{X}>\mathrm{Y}$. When you pay attention to each pair of alternatives, only the relation between $X$ and $Y$ is obviously right, the others may not when considering special attribute. So, this method neglects the same thing as the above method.

For the Lexicography method, if the first attribute Range is the most important attribute, then the value for alternative $X / Y / Z$ are $1,0.8$ and 0.5 , respectively. So the order of alternatives is $X>Y>Z$. This method would neglect the weight of the attribute. For example, if the weight of the first attribute "Range" is 0.3 , the weight of attributes "Accuracy" and "Vulnerability" are both 0.25 . Then, alternative $\mathrm{Z}$ would exceed $\mathrm{X}$ and reverse to the best one.

For the additive weighting method, if we set the attribute weight be $(0.05,0.1,0.1,0.4,0.15,0.2)$, the order result would be $X>Z>Y$. If the attribute weight changes tiny to (0.04, $0.1,0.1,0.4,0.16,0.2)$, the order result will be different. This rank method largely relies on the attribute weight. If attribute weight changes little, you would make a different decision and take another alternative as the best one.

For the non-metric scaling method, when you locate an ideal object $(1,1,1,1,1,1,1)$ in the attribute space, the distance between alternative $X / Y / Z$ and ideal object are $0.5283,1.4824,1.0058$. Alternative $X$ is close to the ideal object. And the order result is $\mathrm{X}>\mathrm{Z}>\mathrm{Y}$. This method also neglect the advantage of alternative $Z$ in "Accuracy" and "Vulnerability". If decision maker takes these two attribute as an important one, then alternative $Z$ would be the optimal.

Each method has its special application environment. None of these methods can be taken as a universal method. To construct a universal method for MADM problem, in next section, an analyzing method different from every method introduced in this section will be proposed based on the Possibility degree. In order to understand the possibility-priority degree analyzing method easily, we consider the MADM problem from easy to general. Section 3 is to consider a MADM problem where the attributes are in same importance. Section 4 is to consider a MADM problem in general condition, considering different attribute importance.

\section{Possibility Priority Degree for Special MADM Problem}

In this section, we considering a special multiple attribute decision making problem, whose attributes take the same importance. Suppose there are $m$ alternatives: $x_{1}, x_{2}, \ldots, x_{m}, n$ attributes: $u_{1}, u_{2}, \ldots, u_{n}$. The MADM information can be shown in Table 7 .

Table 7. A MADM problem.

\begin{tabular}{lllll}
\hline & $\mathbf{u}_{1}$ & $\mathbf{u}_{2}$ & $\ldots$ & $\mathbf{u}_{\mathbf{n}}$ \\
\hline $\mathrm{x}_{1}$ & $\mathrm{a}_{11}$ & $\mathrm{a}_{12}$ & $\ldots$ & $\mathrm{a}_{1 \mathrm{n}}$ \\
$\mathrm{x}_{2}$ & $\mathrm{a}_{21}$ & $\mathrm{a}_{22}$ & $\ldots$ & $\mathrm{a}_{2 \mathrm{n}}$ \\
$\ldots$ & & & $\ldots$ & $\ldots$ \\
$\mathrm{x}_{\mathrm{m}}$ & $\mathrm{a}_{\mathrm{m} 1}$ & $\mathrm{a}_{\mathrm{m} 2}$ & $\ldots$ & $\mathrm{a}_{\mathrm{mn}}$ \\
\hline
\end{tabular}

Definition 1: For an attribute $u_{j}(j=1,2, \ldots, n)$ and any two alternatives $x_{i}$ and $x_{k}$ (Their attribute information for $u_{j}$ is $a_{i j}$ and $a_{k j}$, respectively; $i, k=1,2, \ldots, m$ ), if $a_{i j}>a_{k j}$, then alternative $x_{i}$ is success to $x_{k}$ for attribute 
$u_{j}$, we call that the priority degree (P) of $x_{i}$ success to $x_{k}$ for $u_{j}$ is 1 , the similar degree $(\mathrm{S})$ of $x_{i}$ similar to $x_{k}$ for $u_{j}$ is 0 , and the priority degree (P) of $x_{k}$ success to $x_{i}$ for $u_{j}$ is 0 . If $a_{i j}=a_{k j}$, then alternative $x_{i}$ is similar to $x_{k}$ for attribute $u_{j}$, we call that the priority degree of $x_{i}$ success to $x_{k}$ for $u_{j}$ is 0 , the similar degree of $x_{i}$ similar to $x_{k}$ for $u_{j}$ is 1 , and the priority degree of $x_{k}$ success to $x_{i}$ for $u_{j}$ is 0 . If $a_{i j}<a_{k j}$, then alternative $x_{k}$ is success to $x_{i}$ for attribute $u_{j}$, we call that the priority degree of $x_{i}$ success to $x_{k}$ for $u_{j}$ is 0 , the similar degree of $x_{i}$ similar to $x_{k}$ for $u_{j}$ is 0 , and the priority degree of $x_{k}$ success to $x_{i}$ for $u_{j}$ is 1 . We note that

(i) $\operatorname{Pr}\left\{x_{i}^{j} \succ x_{k}^{j}\right\}=1, S\left\{x_{i}^{j} \sim x_{k}^{j}\right\}=0, \operatorname{Pr}\left\{x_{k}^{j} \succ x_{i}^{j}\right\}=0$, if $a_{i j}>a_{k j}$,

(ii) $\operatorname{Pr}\left\{x_{i}^{j} \succ x_{k}^{j}\right\}=0, S\left\{x_{i}^{j} \sim x_{k}^{j}\right\}=1, \operatorname{Pr}\left\{x_{k}^{j} \succ x_{i}^{j}\right\}=0$, if $a_{i j}=a_{k j}$,

(iii) $\operatorname{Pr}\left\{x_{i}^{j} \succ x_{k}^{j}\right\}=0, S\left\{x_{i}^{j} \sim x_{k}^{j}\right\}=0, \operatorname{Pr}\left\{x_{k}^{j} \succ x_{i}^{j}\right\}=1$, if $a_{i j}<a_{k j}$,

Where ' $\succ^{\prime},{ }^{\prime} \sim^{\prime}$ are priority and similar, respectively (See more from "Multiple criteria decision making"[16]), $\operatorname{Pr}\{\cdot\}$ is the priority degree and $S\{\cdot\}$ is the similar degree.

Property 1: In a MADM problem, for all $i, k, u=1,2, \ldots, m ; j=1,2, \ldots, n$,

(i) $0 \leq \operatorname{Pr}\left\{x_{i}^{j} \succ x_{k}^{j}\right\} \leq 1,0 \leq S\left\{x_{i}^{j} \sim x_{k}^{j}\right\} \leq 1$,

(ii) $\operatorname{Pr}\left\{x_{i}^{j} \succ x_{k}^{j}\right\}+S\left\{x_{i}^{j} \sim x_{k}^{j}\right\}+\operatorname{Pr}\left\{x_{k}^{j} \succ x_{i}^{j}\right\}=1$,

(iii) If $\operatorname{Pr}\left\{x_{i}^{j} \succ x_{k}^{j}\right\}=1, \operatorname{Pr}\left\{x_{k}^{j} \succ x_{u}^{j}\right\}=1$, then $\operatorname{Pr}\left\{x_{i}^{j} \succ x_{u}^{j}\right\}=1$,

(iv) If $\operatorname{Pr}\left\{x_{i}^{j} \sim x_{k}^{j}\right\}=1, \operatorname{Pr}\left\{x_{k}^{j} \sim x_{u}^{j}\right\}=1$, then
$\operatorname{Pr}\left\{x_{i}^{j} \sim x_{u}^{j}\right\}=1$

These properties in Property 1 can be illustrated as follows.

Property 1 (i) means the range of priority degree and similar degree. If $a_{i j}>a_{k j}$, then $\operatorname{Pr}\left\{x_{i}^{j} \succ x_{k}^{j}\right\}=1$. Else $\operatorname{Pr}\left\{x_{i}^{j} \succ x_{k}^{j}\right\}=0$. So, $0 \leq \operatorname{Pr}\left\{x_{i}^{j} \succ x_{k}^{j}\right\} \leq 1$. In the same way, we can get that $0 \leq S\left\{x_{i}^{j} \sim x_{k}^{j}\right\} \leq 1$.

Property 1 (ii) shows that the relation between the attribute value of alternative $x_{i}$ and that of $x_{k}$ is certain. Whatever the relation is, only one of the three relations $\operatorname{Pr}\left\{x_{i}^{j} \succ x_{k}^{j}\right\}, S\left\{x_{i}^{j} \sim x_{k}^{j}\right\}, \operatorname{Pr}\left\{x_{k}^{j} \succ x_{i}^{j}\right\}$ takes 1, and the other two relations takes 0 . So, these three sum to 1 .

Property 1 (iii) shows the transitivity of priority degree. On a certain attribute $u_{j}$, if alternative $x_{i}$ is success to $x_{k}$ $\left(\operatorname{Pr}\left\{x_{i}^{j} \succ x_{k}^{j}\right\}=1\right)$ and $x_{k}$ is success to $x_{u}$ $\left(\operatorname{Pr}\left\{x_{k}^{j} \succ x_{u}^{j}\right\}=1\right)$, then alternative $x_{i}$ is success to $x_{u}$ $\left(\operatorname{Pr}\left\{x_{i}^{j} \succ x_{u}^{j}\right\}=1\right)$.

Property 1 (iv) shows the transitivity of similar degree. On a certain attribute $u_{j}$, if alternative $x_{i}$ is similar to $x_{k}$ $\left(\operatorname{Pr}\left\{x_{i}^{j} \sim x_{k}^{j}\right\}=1\right)$ and $x_{k}$ is similar to $x_{u}$ $\left(\operatorname{Pr}\left\{x_{k}^{j} \sim x_{u}^{j}\right\}=1\right)$, then alternative $x_{i}$ is similar to $x_{u}$ $\left(\operatorname{Pr}\left\{x_{i}^{j} \sim x_{u}^{j}\right\}=1\right)$.

For simply noting the Possibility, we simplify the note of $\operatorname{Pr}\left\{x_{i}^{j} \succ x_{k}^{j}\right\}=1, S\left\{x_{i}^{j} \sim x_{k}^{j}\right\}=0$ as $x_{i}^{j} \succ_{100 \%} x_{k}^{j}, x_{i}^{j} \sim_{0 \%} x_{k}^{j}$.

Example 2 Take the example in Example 1 for example, the normalized decision information shows in Table 8 .

Table 8. Comparable Numerical Values for the Problem.

\begin{tabular}{lllllll}
\hline & Range (n mi) & Delivery time (hr) & Total yield (MT) & Accuracy & Vulnerability & Payload delivery flexibility \\
X & 1 & 1 & 1 & 0.7143 & 0.5556 & 1 \\
$\mathrm{Y}$ & 0.8 & 0.1 & 0.5 & 0.4286 & 0.3333 & 0.4286 \\
$\mathrm{Z}$ & 0.5 & 0.2 & 0.8 & 1 & 1 & 0.7143 \\
\hline
\end{tabular}

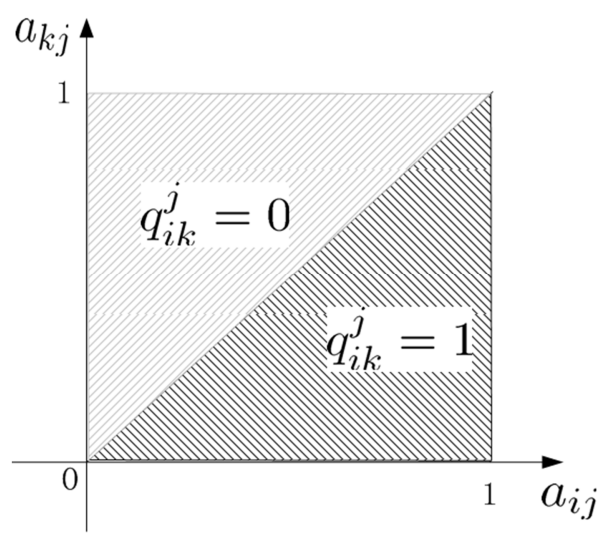

Figure 1. Relations among $a_{i j,} a_{k j}$ and $q_{i k}^{j}$

Random choose one attribute from RangelDelivery time $\backslash$ Total yield $\backslash$ Accuracy $\backslash$ Vulnerability $\backslash$ Payload delivery flexibility as an example. We take Accuracy. On this attribute, these relations can get.

$$
\begin{gathered}
\operatorname{Pr}=\left[\begin{array}{ccc}
X \succ_{0 \%} X & X \succ_{100 \%} Y & X \succ_{0 \%} Z \\
Y \succ_{0 \%} X & Y \succ_{0 \%} Y & Y \succ_{0 \%} Z \\
Z \succ_{100 \%} X & Z \succ_{100 \%} Y & Z \succ_{0 \%} Z
\end{array}\right], \\
S=\left[\begin{array}{ccc}
X \sim_{100 \%} X & X \sim_{0 \%} Y & X \sim_{0 \%} Z \\
Y \sim_{0 \%} X & Y \sim_{100 \%} Y & Y \sim_{0 \%} Z \\
Z \sim_{0 \%} X & Z \sim_{0 \%} Y & Z \sim_{100 \%} Z
\end{array}\right] .
\end{gathered}
$$

It means that on accuracy, alternative $X$ is 100 percent success to $\mathrm{Y}$. $\mathrm{Z}$ is 100 percent success to $\mathrm{X}$. And $\mathrm{Z}$ is 100 percent success to $Y$. They are only 100 percent similar to themselves, separately.

To compare two alternatives, we take a binary variable $q_{i k}^{j}(i, k=1,2, \ldots, m ; j=1,2, \ldots, n)$, which respect the relation between alternative $x_{i}$ and $x_{k}$ on attribute $u_{j}$, if alternative $x_{i}$ on attribute $u_{j}$ is 100 percent priority to $x_{k}$, then $q_{i k}^{j}=q^{j}\left(x_{i}, x_{k}\right)=1$, else, $q_{i k}^{j}=q^{j}\left(x_{i}, x_{k}\right)=0$, where 


$$
q_{i k}^{j}=q^{j}\left(x_{i}, x_{k}\right)= \begin{cases}1, & \text { if } x_{i}^{j} \succ_{100 \%} x_{k}^{j}, \quad i, k=1,2, \ldots, m ; j=1,2, \ldots, n . \\ 0, & \text { else. }\end{cases}
$$

With the binary variable $q_{i k}^{j}$, the attribute, on which alternative $x_{i}$ is 100 percent success to $x_{k}$, can be count that

$$
\operatorname{Count}\left(x_{i} \succ_{100 \%} x_{k}\right)=\sum_{j=1}^{n} q_{i k}^{j}, i, j=1,2, \ldots, m \text {. }
$$

Then, the attribute, on which alternative $x_{i}$ is 100 percent similar to $x_{k}$, can generate that

$$
\operatorname{Count}\left(x_{i} \sim_{100 \%} x_{k}\right)=n-\operatorname{Count}\left(x_{i} \succ_{100 \%} x_{k}\right)-\operatorname{Count}\left(x_{k} \succ_{100 \%} x_{i}\right), i, j=1,2, \ldots, m .
$$

Definition 2: For any two alternatives $x_{i}$ and $x_{k}$ (Their attribute information for $u_{j}(j=1,2, \ldots, n)$ is $a_{i j}$ and $a_{k j}$, respectively; $i, k=1,2, \ldots, m)$,

(i) The Possibility of $x_{i}$ is 100 percent success to $x_{k}$ is that

$$
P\left\{x_{i} \succ_{100 \%} x_{k}\right\}=\frac{\operatorname{Count}\left(x_{i} \succ_{100 \%} x_{k}\right)}{n}, i, k=1,2, \ldots, m .
$$

(ii) The Possibility of $x_{i}$ is 100 percent similar to $x_{k}$ is that

$$
P\left\{x_{i} \sim_{100 \%} x_{k}\right\}=\frac{n-\operatorname{Count}\left(x_{i} \succ_{100 \%} x_{k}\right)-\operatorname{Count}\left(x_{k} \succ_{100 \%} x_{i}\right)}{n}, i, k=1,2, \ldots, m .
$$

where $P\{\cdot\}$ is Possibility degree. $P\left\{x_{i} \succ_{100 \%} x_{k}\right\}$ is the Possibility Priority degree (PPD). $P\left\{x_{i} \sim_{100 \%} x_{k}\right\}$ is the Possibility Similar degree.

Property 2: For any three alternatives $x_{i}, x_{k}$ and $x_{u}(i, k, u=1,2, \ldots, m)$,

(i) $0 \leq P\left\{x_{i} \succ_{100 \%} x_{k}\right\} \leq 1,0 \leq P\left\{x_{i} \sim_{100 \%} x_{k}\right\} \leq 1, i, k=1,2, \ldots, m$,

(ii) $P\left\{x_{i} \succ_{100 \%} x_{k}\right\}+P\left\{x_{i} \sim_{100 \%} x_{k}\right\}+P\left\{x_{k} \succ_{100 \%} x_{i}\right\}=1, i, k=1,2, \ldots, m$,

(iii) If $P\left\{x_{i} \succ_{100 \%} x_{k}\right\}=1, P\left\{x_{k} \succ_{100 \%} x_{u}\right\}=1$, then $P\left\{x_{i} \succ_{100 \%} x_{u}\right\}=1$,

(iv) If $P\left\{x_{i} \sim_{100 \%} x_{k}\right\}=1, P\left\{x_{k} \sim_{100 \%} x_{u}\right\}=1$, then $P\left\{x_{i} \sim_{100 \%} x_{u}\right\}=1$.

Property 2 (i) means that the Possibility of alternative $x_{i}$ 100 percent success to $x_{j}$ is between 0 and 1 . If every attribute value of alternative $x_{i}$ is better than that of $x_{j}$, then the Possibility of alternative $x_{i} 100$ percent success to $x_{j}$ is 1 . If every attribute value of alternative $x_{i}$ is not better than that of $x_{j}$, then the Possibility of alternative $x_{i} 100$ percent success to $x_{j}$ is 0 . If some attributes' value of alternative $x_{i}$ is better than that of $x_{j}$ while some attributes not, then the Possibility of alternative $x_{i} 100$ percent success to $x_{j}$ is between 0 and 1 . And the Possibility of alternative $x_{i} 100$ percent similar to $x_{j}$ is between 0 and 1 . If every attribute value of alternative $x_{i}$ is equal to that of $x_{j}$, then the Possibility of alternative $x_{i} 100$ percent similar to $x_{j}$ is 1 . If every attribute value of alternative $x_{i}$ is not equal to that of $x_{j}$, then the Possibility of alternative $x_{i} 100$ percent similar to $x_{j}$ is 0 . If some attributes' value of alternative $x_{i}$ is equal to that of $x_{j}$ while some attributes not, then the Possibility of alternative $x_{i} 100$ percent similar to $x_{j}$ is between 0 and 1 .

Property 2 (ii) means that for any two alternatives $x_{i}$ and $x_{k}$, summation of the Possibility of alternative $x_{i} 100$ percent success to $x_{j}, x_{i} 100$ percent similar to $x_{j}$, and $x_{k} 100$ percent success to $x_{i}$ is 1 .

Property 2 (iii) means that for any three alternatives $x_{i}, x_{k}, x_{u}$, if alternative $x_{i}$ is 100 percent success to $x_{k}$, and alternative $x_{k}$ is 100 percent success to $x_{u}$, then alternative $x_{i}$ is 100 percent success to $x_{u}$.

Property 2 (iv) means that for any three alternatives $x_{i}, x_{k}, x_{u}$, if alternative $x_{i}$ is 100 percent similar to $x_{k}$, and alternative $x_{k}$ is 100 percent similar to $x_{u}$, then alternative $x_{i}$ is 100 percent similar to $x_{u}$.

Example 3 Take the example in Example 1 for example, the normalized decision information shows in Table 9.

Table 9. Comparable Numerical Values for the Problem.

\begin{tabular}{lllllll}
\hline & Range (n mi) & Delivery time (hr) & Total yield (MT) & Accuracy & Vulnerability & Payload delivery flexibility \\
\hline X & 1 & 1 & 1 & 0.7143 & 0.5556 & 1 \\
Y & 0.8 & 0.1 & 0.5 & 0.4286 & 0.3333 & 0.4286 \\
Z & 0.5 & 0.2 & 0.8 & 1 & 1 & 0.7143 \\
\hline
\end{tabular}

For attribute Range, the priority and similar degree can generate that

$$
P=\left[\begin{array}{ccc}
X \succ_{0 \%} X & X \succ_{100 \%} Y & X \succ_{100 \%} Z \\
Y \succ_{0 \%} X & Y \succ_{0 \%} Y & Y \succ_{100 \%} Z \\
Z \succ_{0 \%} X & Z \succ_{0 \%} Y & Z \succ_{0 \%} Z
\end{array}\right], S=\left[\begin{array}{ccc}
X \sim_{100 \%} X & X \sim_{0 \%} Y & X \sim_{0 \%} Z \\
Y \sim_{0 \%} X & Y \sim_{100 \%} Y & Y \sim_{0 \%} Z \\
Z \sim_{0 \%} X & Z \sim_{0 \%} Y & Z \sim_{100 \%} Z
\end{array}\right] .
$$


For attribute Delivery time, the priority and similar degree can generate that

$$
P=\left[\begin{array}{ccc}
X \succ_{0 \%} X & X \succ_{100 \%} Y & X \succ_{100 \%} Z \\
Y \succ_{0 \%} X & Y \succ_{0 \%} Y & Y \succ_{0 \%} Z \\
Z \succ_{0 \%} X & Z \succ_{100 \%} Y & Z \succ_{0 \%} Z
\end{array}\right], S=\left[\begin{array}{ccc}
X \sim_{100 \%} X & X \sim_{0 \%} Y & X \sim_{0 \%} Z \\
Y \sim_{0 \%} X & Y \sim_{100 \%} Y & Y \sim_{0 \%} Z \\
Z \sim_{0 \%} X & Z \sim_{0 \%} Y & Z \sim_{100 \%} Z
\end{array}\right] .
$$

For attribute Total yield, the priority and similar degree can generate that

$$
P=\left[\begin{array}{ccc}
X \succ_{0 \%} X & X \succ_{100 \%} Y & X \succ_{100 \%} Z \\
Y \succ_{0 \%} X & Y \succ_{0 \%} Y & Y \succ_{0 \%} Z \\
Z \succ_{0 \%} X & Z \succ_{100 \%} Y & Z \succ_{0 \%} Z
\end{array}\right], S=\left[\begin{array}{ccc}
X \sim_{100 \%} X & X \sim_{0 \%} Y & X \sim_{0 \%} Z \\
Y \sim_{0 \%} X & Y \sim_{100 \%} Y & Y \sim_{0 \%} Z \\
Z \sim_{0 \%} X & Z \sim_{0 \%} Y & Z \sim_{100 \%} Z
\end{array}\right] .
$$

For attribute Accuracy, the priority and similar degree can generate that

$$
P=\left[\begin{array}{ccc}
X \succ_{0 \%} X & X \succ_{100 \%} Y & X \succ_{0 \%} Z \\
Y \succ_{0 \%} X & Y \succ_{0 \%} Y & Y \succ_{0 \%} Z \\
Z \succ_{100 \%} X & Z \succ_{100 \%} Y & Z \succ_{0 \%} Z
\end{array}\right], S=\left[\begin{array}{ccc}
X \sim_{100 \%} X & X \sim_{0 \%} Y & X \sim_{0 \%} Z \\
Y \sim_{0 \%} X & Y \sim_{100 \%} Y & Y \sim_{0 \%} Z \\
Z \sim_{0 \%} X & Z \sim_{0 \%} Y & Z \sim_{100 \%} Z
\end{array}\right]
$$

For attribute Vulnerability, the priority and similar degree can generate that

$$
P=\left[\begin{array}{ccc}
X \succ_{0 \%} X & X \succ_{100 \%} Y & X \succ_{0 \%} Z \\
Y \succ_{0 \%} X & Y \succ_{0 \%} Y & Y \succ_{0 \%} Z \\
Z \succ_{100 \%} X & Z \succ_{100 \%} Y & Z \succ_{0 \%} Z
\end{array}\right], S=\left[\begin{array}{ccc}
X \sim_{100 \%} X & X \sim_{0 \%} Y & X \sim_{0 \%} Z \\
Y \sim_{0 \%} X & Y \sim_{100 \%} Y & Y \sim_{0 \%} Z \\
Z \sim_{0 \%} X & Z \sim_{0 \%} Y & Z \sim_{100 \%} Z
\end{array}\right]
$$

For attribute Payload delivery flexibility, the priority and similar degree can generate that

$$
P=\left[\begin{array}{ccc}
X \succ_{0 \%} X & X \succ_{100 \%} Y & X \succ_{100 \%} Z \\
Y \succ_{0 \%} X & Y \succ_{0 \%} Y & Y \succ_{0 \%} Z \\
Z \succ_{0 \%} X & Z \succ_{100 \%} Y & Z \succ_{0 \%} Z
\end{array}\right], S=\left[\begin{array}{ccc}
X \sim_{100 \%} X & X \sim_{0 \%} Y & X \sim_{0 \%} Z \\
Y \sim_{0 \%} X & Y \sim_{100 \%} Y & Y \sim_{0 \%} Z \\
Z \sim_{0 \%} X & Z \sim_{0 \%} Y & Z \sim_{100 \%} Z
\end{array}\right] .
$$

Then, the Possibility of one alternative is 100 percent success to the other in each pair of alternative $\mathrm{X}, \mathrm{Y}, \mathrm{Z}$ can be calculated that

$$
\left[\begin{array}{lll}
P\left\{X \succ_{100 \%} X\right\} & P\left\{X \succ_{100 \%} Y\right\} & P\left\{X \succ_{100 \%} Z\right\} \\
P\left\{Y \succ_{100 \%} X\right\} & P\left\{Y \succ_{100 \%} Y\right\} & P\left\{Y \succ_{100 \%} Z\right\} \\
P\left\{Z \succ_{100 \%} X\right\} & P\left\{Z \succ_{100 \%} Y\right\} & P\left\{Z \succ_{100 \%} Z\right\}
\end{array}\right]=\left[\begin{array}{ccc}
0 \% & 100 \% & 67 \% \\
0 \% & 0 \% & 17 \% \\
33 \% & 83 \% & 0 \%
\end{array}\right]
$$

The possibility of one alternative is 100 percent similar to the other in each pair of alternative $\mathrm{X}, \mathrm{Y}, \mathrm{Z}$ can be calculated that

$$
\left[\begin{array}{lll}
P\left\{X \sim_{100 \%} X\right\} & P\left\{X \sim_{100 \%} Y\right\} & P\left\{X \sim_{100 \%} Z\right\} \\
P\left\{Y \sim_{100 \%} X\right\} & P\left\{Y \sim_{100 \%} Y\right\} & P\left\{Y \sim_{100 \%} Z\right\} \\
P\left\{Z \sim_{100 \%} X\right\} & P\left\{Z \sim_{100 \%} Y\right\} & P\left\{Z \sim_{100 \%} Z\right\}
\end{array}\right]=\left[\begin{array}{ccc}
100 \% & 0 \% & 0 \% \\
0 \% & 100 \% & 0 \% \\
0 \% & 0 \% & 100 \%
\end{array}\right]
$$

From the possibility of priority degree, we can see that the Possibility of alternative $\mathrm{X} 100$ percent success to $\mathrm{Y}$ is $100 \%$. It means that $\mathrm{X}$ is the best choice when you choose one from $\mathrm{X}$ and $\mathrm{Y}$.

The Possibility of alternative $\mathrm{X} 100$ percent success to $\mathrm{Z}$ is $67 \%$. It means that the Possibility of $X$ better than $Z$ is $67 \%$. It do not means that $X$ is the best when you choose choice one from $\mathrm{X}$ and $\mathrm{Z}$. While you choose $\mathrm{X}$ from $\mathrm{X}$ and $\mathrm{Z}$, the risk of this decision exist. So when do decision making, decision maker should be aware of the risk.

The Possibility of alternative $\mathrm{Y} 100$ percent success to $\mathrm{Z}$ is $17 \%$. It means that the Possibility of $Y$ better than $Z$ is $17 \%$. It does not mean that $Z$ is the best choice. Alternative $Y$ still has 17 percent chance better than $Z$.

The Possibility of alternative $Z 100$ percent success to $X$ is $33 \%$. It means that the Possibility of $Z$ better than $X$ is $33 \%$. It does not mean that $\mathrm{X}$ is the best choice. Alternative $\mathrm{Z}$ still has
33 percent chance better than $X$.

The Possibility of alternative $\mathrm{Z} 100$ percent success to $\mathrm{Y}$ is $83 \%$. It means that the Possibility of $Z$ better than $Y$ is $83 \%$. It means that $\mathrm{Z}$ is the best choice when choose one from $\mathrm{Y}$ and $\mathrm{Z}$. But the risk still exists.

The Possibility of alternative X 100 percent success to X, Y 100 percent success to $\mathrm{Y}, \mathrm{Z} 100$ percent success to $\mathrm{Z}$ and $\mathrm{Y}$ 100 percent success to $X$ are $0 \%$. Alternative $X, Y, Z$ success to itself is impossible. And $\mathrm{Z}$ success to $\mathrm{Y}$ is also impossible.

From the possibility of similar degree, we can see that only the possibility of alternative $\mathrm{X}, \mathrm{Y}, \mathrm{Z}$ similar to itself is $100 \%$. The possibility degree of they similar to each other is 0.

So, a decision maker may take the alternative $\mathrm{X}$ as the best one. While choosing alternative $\mathrm{X}$, decision maker should aware of the risk still existing. 


\section{Possibility Priority Degree for General MADM Problem}

In this section, a general MADM problem with different attribute weight is taken into account.

With the traditional MADM method, there are a mount of methods to generate the attribute weight, including integrated weights method, objective weight method and subjective weight method [17]. According to the decision maker, each of them can be used to generate the attribute weight.

Suppose there are $m$ alternatives: $x_{1}, x_{2}, \ldots, x_{m}, n$ attributes: $u_{1}, u_{2}, \ldots, u_{n}$. And the attribute weight can get that $w_{1}, w_{2}, \ldots, w_{n}$, where

$$
\sum_{i=1}^{n} w_{j}=1, w_{j}>0, j=1,2, \ldots, n .
$$

Let

$$
J_{i k}^{\succ}=\left\{u_{j} \mid x_{i}^{j} \succ_{100 \%} x_{k}^{j}, j=1,2, \ldots, n .\right\}, i, k=1,2, \ldots, m .
$$

be a subset of attributes, on which alternative $x_{i}$ is 100 percent success to $x_{k}$ and

$$
J_{i k}^{\sim}=\left\{u_{j} \mid x_{i}^{j} \sim_{100 \%} x_{k}^{j}, j=1,2, \ldots, n .\right\}, i, k=1,2, \ldots, m .
$$

be a subset of attributes, on which alternative $x_{i}$ is 100 percent similar to $x_{k}$.

Definition3: Suppose the attribute weights are $w_{1}, w_{2}, \ldots, w_{n}$. For any two alternatives $x_{i}$ and $x_{k}$ (Their attribute information for $u_{j}(j=1,2, \ldots, n)$ is $a_{i j}$ and $a_{k j}$, respectively; $i, k=1,2, \ldots, m)$,

(i) The Possibility of $x_{i}$ is 100 percent success to $x_{k}$ is that

$$
P\left\{x_{i} \succ_{100 \%} x_{k}\right\}=\sum_{u_{j} \in J_{i k}^{\succ}} w_{j}, i, k=1,2, \ldots, m .
$$

(ii) The Possibility of $x_{i}$ is 100 percent similar to $x_{k}$ is that

$$
P\left\{x_{i} \sim_{100 \%} x_{k}\right\}=\sum_{u_{j} \in J_{i k}} w_{j}, i, k=1,2, \ldots, m .
$$

Where $P\{\cdot\}$ is Possibility (Access more knowledge about Possibility form "Possibility: theory and examples" [18]). $P\left\{x_{i} \succ_{100 \%} x_{k}\right\}$ is the Possibility Priority degree (PPD). $P\left\{x_{i} \sim_{100 \%} x_{k}\right\}$ is the Possibility Similar degree.

Mark 2: The possibility of priority degree and similar degree in Definition 3 hold each property in Property 2.

Example 4 Take the example in Example 1 for example, the normalized decision information shows in Table 10.

Table 10. Comparable Numerical Values for the Problem.

\begin{tabular}{lllllll}
\hline & Range (n mi) & Delivery time (hr) & Total yield (MT) & Accuracy & Vulnerability & Payload delivery flexibility \\
\hline X & 1 & 1 & 1 & 0.7143 & 0.5556 & 1 \\
$\mathrm{Y}$ & 0.8 & 0.1 & 0.5 & 0.4286 & 0.3333 & 0.4286 \\
$\mathrm{Z}$ & 0.5 & 0.2 & 0.8 & 1 & 1 & 0.7143 \\
\hline
\end{tabular}

Suppose the attribute weights are

$$
w_{1}=0.15, w_{2}=0.05, w_{3}=0.1, w_{4}=0.4, w_{5}=0.2, w_{6}=0.1 .
$$

For attribute Range, the priority and similar degree can generate that

$$
P=\left[\begin{array}{ccc}
X \succ_{0 \%} X & X \succ_{100 \%} Y & X \succ_{100 \%} Z \\
Y \succ_{0 \%} X & Y \succ_{0 \%} Y & Y \succ_{100 \%} Z \\
Z \succ_{0 \%} X & Z \succ_{0 \%} Y & Z \succ_{0 \%} Z
\end{array}\right], S=\left[\begin{array}{ccc}
X \sim_{100 \%} X & X \sim_{0 \%} Y & X \sim_{0 \%} Z \\
Y \sim_{0 \%} X & Y \sim_{100 \%} Y & Y \sim_{0 \%} Z \\
Z \sim_{0 \%} X & Z \sim_{0 \%} Y & Z \sim_{100 \%} Z
\end{array}\right] .
$$

For attribute Delivery time, the priority and similar degree can generate that

$$
P=\left[\begin{array}{ccc}
X \succ_{0 \%} X & X \succ_{100 \%} Y & X \succ_{100 \%} Z \\
Y \succ_{0 \%} X & Y \succ_{0 \%} Y & Y \succ_{0 \%} Z \\
Z \succ_{0 \%} X & Z \succ_{100 \%} Y & Z \succ_{0 \%} Z
\end{array}\right], S=\left[\begin{array}{ccc}
X \sim_{100 \%} X & X \sim_{0 \%} Y & X \sim_{0 \%} Z \\
Y \sim_{0 \%} X & Y \sim_{100 \%} Y & Y \sim_{0 \%} Z \\
Z \sim_{0 \%} X & Z \sim_{0 \%} Y & Z \sim_{100 \%} Z
\end{array}\right] .
$$

For attribute Total yield, the priority and similar degree can generate that

$$
P=\left[\begin{array}{ccc}
X \succ_{0 \%} X & X \succ_{100 \%} Y & X \succ_{100 \%} Z \\
Y \succ_{0 \%} X & Y \succ_{0 \%} Y & Y \succ_{0 \%} Z \\
Z \succ_{0 \%} X & Z \succ_{100 \%} Y & Z \succ_{0 \%} Z
\end{array}\right], S=\left[\begin{array}{ccc}
X \sim_{100 \%} X & X \sim_{0 \%} Y & X \sim_{0 \%} Z \\
Y \sim_{0 \%} X & Y \sim_{100 \%} Y & Y \sim_{0 \%} Z \\
Z \sim_{0 \%} X & Z \sim_{0 \%} Y & Z \sim_{100 \%} Z
\end{array}\right] .
$$

For attribute Accuracy, the priority and similar degree can generate that

$$
P=\left[\begin{array}{ccc}
X \succ_{0 \%} X & X \succ_{100 \%} Y & X \succ_{0 \%} Z \\
Y \succ_{0 \%} X & Y \succ_{0 \%} Y & Y \succ_{0 \%} Z \\
Z \succ_{100 \%} X & Z \succ_{100 \%} Y & Z \succ_{0 \%} Z
\end{array}\right], S=\left[\begin{array}{ccc}
X \sim_{100 \%} X & X \sim_{0 \%} Y & X \sim_{0 \%} Z \\
Y \sim_{0 \%} X & Y \sim_{100 \%} Y & Y \sim_{0 \%} Z \\
Z \sim_{0 \%} X & Z \sim_{0 \%} Y & Z \sim_{100 \%} Z
\end{array}\right]
$$

For attribute Vulnerability, the priority and similar degree can generate that 


$$
P=\left[\begin{array}{ccc}
X \succ_{0 \%} X & X \succ_{100 \%} Y & X \succ_{0 \%} Z \\
Y \succ_{0 \%} X & Y \succ_{0 \%} Y & Y \succ_{0 \%} Z \\
Z \succ_{100 \%} X & Z \succ_{100 \%} Y & Z \succ_{0 \%} Z
\end{array}\right], S=\left[\begin{array}{ccc}
X \sim_{100 \%} X & X \sim_{0 \%} Y & X \sim_{0 \%} Z \\
Y \sim_{0 \%} X & Y \sim_{100 \%} Y & Y \sim_{0 \%} Z \\
Z \sim_{0 \%} X & Z \sim_{0 \%} Y & Z \sim_{100 \%} Z
\end{array}\right]
$$

For attribute Payload delivery flexibility, the priority and similar degree can generate that

$$
P=\left[\begin{array}{ccc}
X \succ_{0 \%} X & X \succ_{100 \%} Y & X \succ_{100 \%} Z \\
Y \succ_{0 \%} X & Y \succ_{0 \%} Y & Y \succ_{0 \%} Z \\
Z \succ_{0 \%} X & Z \succ_{100 \%} Y & Z \succ_{0 \%} Z
\end{array}\right], S=\left[\begin{array}{ccc}
X \sim_{100 \%} X & X \sim_{0 \%} Y & X \sim_{0 \%} Z \\
Y \sim_{0 \%} X & Y \sim_{100 \%} Y & Y \sim_{0 \%} Z \\
Z \sim_{0 \%} X & Z \sim_{0 \%} Y & Z \sim_{100 \%} Z
\end{array}\right] .
$$

Then, the Possibility of one alternative is 100 percent success to the other in each pair of alternative X, Y, Z can be calculated that

$$
\left[\begin{array}{lll}
P\left\{X \succ_{100 \%} X\right\} & P\left\{X \succ_{100 \%} Y\right\} & P\left\{X \succ_{100 \%} Z\right\} \\
P\left\{Y \succ_{100 \%} X\right\} & P\left\{Y \succ_{100 \%} Y\right\} & P\left\{Y \succ_{100 \%} Z\right\} \\
P\left\{Z \succ_{100 \%} X\right\} & P\left\{Z \succ_{100 \%} Y\right\} & P\left\{Z \succ_{100 \%} Z\right\}
\end{array}\right]=\left[\begin{array}{ccc}
0 \% & 100 \% & 40 \% \\
0 \% & 0 \% & 15 \% \\
60 \% & 85 \% & 0 \%
\end{array}\right]
$$

The possibility of one alternative is 100 percent similar to the other in each pair of alternative $\mathrm{X}, \mathrm{Y}, \mathrm{Z}$ can be calculated that

$$
\left[\begin{array}{lll}
P\left\{X \sim_{100 \%} X\right\} & P\left\{X \sim_{100 \%} Y\right\} & P\left\{X \sim_{100 \%} Z\right\} \\
P\left\{Y \sim_{100 \%} X\right\} & P\left\{Y \sim_{100 \%} Y\right\} & P\left\{Y \sim_{100 \%} Z\right\} \\
P\left\{Z \sim_{100 \%} X\right\} & P\left\{Z \sim_{100 \%} Y\right\} & P\left\{Z \sim_{100 \%} Z\right\}
\end{array}\right]=\left[\begin{array}{ccc}
100 \% & 0 \% & 0 \% \\
0 \% & 100 \% & 0 \% \\
0 \% & 0 \% & 100 \%
\end{array}\right]
$$

From the possibility of priority degree, we can see that the Possibility of alternative X 100 percent success to $\mathrm{Y}$ is $100 \%$. It means that $\mathrm{X}$ is the best choice when you choose one from $\mathrm{X}$ and $\mathrm{Y}$.

The Possibility of alternative $\mathrm{X} 100$ percent success to $\mathrm{Z}$ is $40 \%$. It means that the Possibility of $X$ better than $\mathrm{Z}$ is $40 \%$. It do not means that $\mathrm{X}$ is the worse when you choose choice one from $\mathrm{X}$ and $\mathrm{Z}$. While you give up $\mathrm{X}$, the advantages of $\mathrm{X}$ still exist. So when do decision making, decision maker should be aware of the risk.

The Possibility of alternative $\mathrm{Y} 100$ percent success to $\mathrm{Z}$ is $15 \%$. It means that the Possibility of $Y$ better than $Z$ is $15 \%$. It does not mean that $Z$ is the best choice. Alternative $Y$ still has 15 percent chance better than $Z$.

The Possibility of alternative $\mathrm{Z} 100$ percent success to $\mathrm{X}$ is $60 \%$. It means that the Possibility of $Z$ better than $X$ is $60 \%$. It does not mean that $\mathrm{Z}$ is the best choice. Alternative $\mathrm{X}$ still has 40 percent chance better than $Z$.

The Possibility of alternative $\mathrm{Z} 100$ percent success to $\mathrm{Y}$ is $85 \%$. It means that the Possibility of $Z$ better than $Y$ is $85 \%$. It means that $\mathrm{Z}$ is the best choice when choose one from $\mathrm{Y}$ and $\mathrm{Z}$. But the risk still exists.

The Possibility of alternative $X 100$ percent success to $X$, Y 100 percent success to $Y, Z 100$ percent success to $Z$ and $\mathrm{Y} 100$ percent success to $\mathrm{X}$ are $0 \%$. Alternative $\mathrm{X}, \mathrm{Y}, \mathrm{Z}$ success to itself is impossible. And $\mathrm{Z}$ success to $\mathrm{Y}$ is also impossible.

From the possibility of similar degree, we can see that only the possibility of alternative $\mathrm{X}, \mathrm{Y}, \mathrm{Z}$ similar to itself is $100 \%$. The possibility degree of they similar to each other is 0 .

So, a decision maker may take the alternative $\mathrm{Z}$ as the best one. While choosing alternative $\mathrm{Z}$, decision maker should aware of the risk still existing.

\section{The PPDA Process}

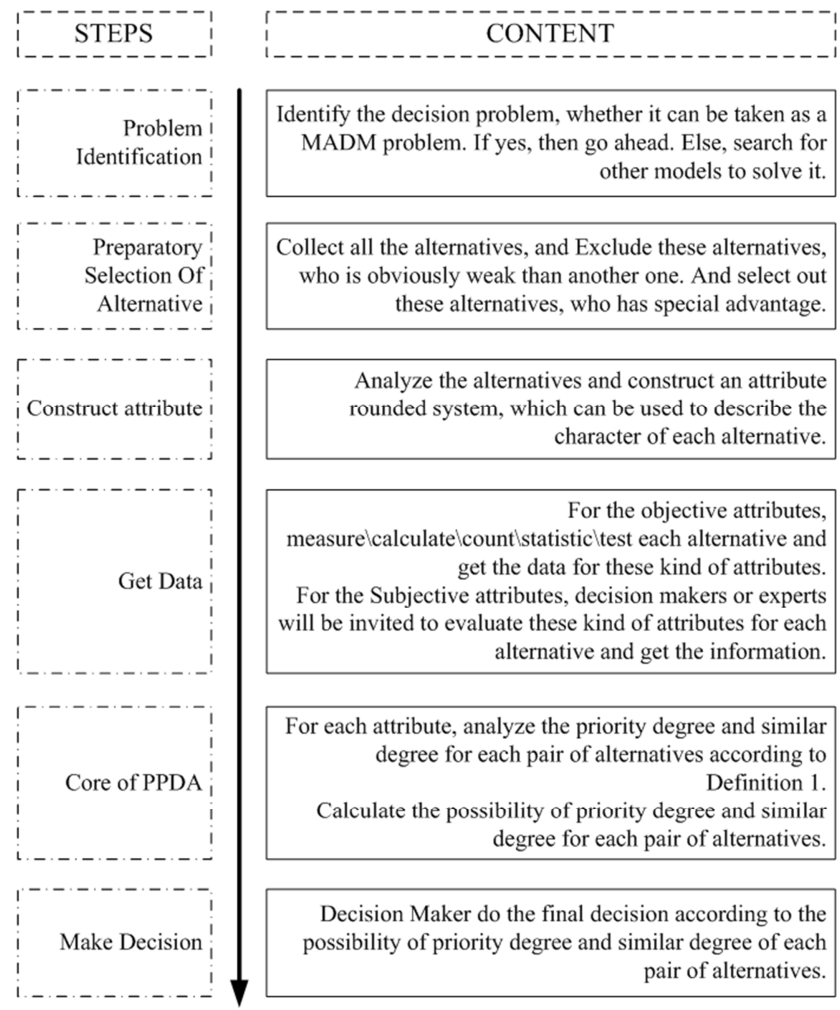

Figure 2. The PPDA process.

\section{Conclusion}

This paper is aim to propose a method for MADM problem, called possibility priority degree analyzing model. To overview the method for MADM problem, in Section 2, The existed methods, including Dominance method, Satisficing 
method, Maximin method, Maximax method, Lexicography method, Additive weighting method and Non-metric scaling method, are introduced and explained by an Example. The result shows that each method has its special application environment. None of the existed methods can be taken as a universal method.

To construct a universal method for MADM problem, in Section 3, a special MADM problem without considering the attribute importance is studied and a possibility priority degree model is proposed. We construct the priority degree and similar degree for two alternatives in a certain attribute. To summarize the relationship between two alternatives in all attributes, a possibility priority degree and possibility similar degree are proposed. With this possibility priority degree, decision maker can easily get the possibility degree of one alternative priority to another. At the same time, decision maker will be aware of the risk when he/she makes decision.

To generate a general MADM model, a MADM problem considering the attribute weights is studied in Section 4. With the integrated weights method, objective weight method or subjective weight method, decision makers can generate the attribute weights easily. In this condition, we propose the possibility priority degree and possibility similar degree model with different attribute weights. And the new possibility priority degree model satisfies the properties in Property 2 . With this possibility priority degree model, the general PPDA process is generated.

Compare the possibility priority degree analyzing process with these existed, there are two main differences of this model:

(1) Return the decision right to decision maker

The host of a decision making is the decision maker. All we can discuss here is to analyze the problem. We have no right to do decision for decision maker. So, you can provide several feasible plans and their related information for decision maker to do final decision. If you provide a certain order of these alternatives, the decision maker is fired. From Table 6, we can see that each of these existed method excepting Dominance method and Satisficing method generates a ranking result finally. Decision maker have no chance to do a choice. With the possibility priority degree analyzing process, we provide a possibility priority degree matrix. Decision maker can get the advantages and disadvantages easily, and then do final decision.

(2) Remind the risk while do decision making

In real word MADM problem, when you decide to choose one and give up another one, there exists the risk hidden in the decision. Except for the group of alternatives which can make decision by the Dominance method, each one in the group of alternatives has both advantages and disadvantages. While choosing one of it, you give up the advantages of another one. It means that the other one may be better than the chosen one in some special condition. So, there are risks when you rank the alternatives. You should let the decision maker be aware of it. From Table 6, we can see that each of these existed method excepting Dominance method and Satisficing method generate a ranking result. It means that the order is the final decision. When suggest the order to decision maker, it means that the order is certain, the order is 100 percent right. Actually, the risk hides on each order. Conversely, the possibility of priority degree shows the possibility of one alternative better than another one. Decision maker can do finally decision with the possibility priority degree. When they do decision, they are aware of the risk of the priority relation.

\section{Acknowledgements}

This research is supported by the Natural Science Foundation of China (Nos.71761001, 71761002) and Guangxi high school innovation team and outstanding scholars plan.

\section{References}

[1] Mac Crimmon, K. R. (1968). Decision making among multiple-attribute alternatives: a survey and consolidated approach (No. RM-4823-ARPA). RAND CORP SANTA MONICA CA.

[2] Fishburn, P. C. (1965). Independence in utility theory with whole product sets. Operations Research, 13 (1), 28-45.

[3] Terry, H. (1963). Comparative evaluation of performance using multiple criteria. Management Science, 9 (3), 431-442.

[4] Papandreou, A. G. (1957). A test of a stochastic theory of choice (Vol. 16). University of California Press.

[5] Churchman, C. W., Ackoff, R. L., \& Arnoff, E. L. (1957). Introduction to operations research.

[6] Aumann, R. J., \& Kruskal, J. B. (1958). The coefficients in an allocation problem. Naval Research Logistics Quarterly, 5 (2), 111-123.

[7] Wohlstetter, A. (1964). Analysis and design of conflict systems. Analysis for military decisions, 103-148.

[8] Simon, H. A. (1955). A behavioral model of rational choice. The quarterly journal of economics, 99-118.

[9] Fishburn, P. C. (1964). Decision and value theory (No. 511.65 F5).

[10] Shepard, R. N. (1962). The analysis of proximities: Multidimensional scaling with an unknown distance function. I Psychometrika, 27 (2), 125-140.

[11] Hwang, C. L., Yoon, K. (1981). Multiple Attribute Decision Making, Springer-Verlag, Berlin.

[12] Milani, A. S., Shanian, A., Madoliat, R., \& Nemes, J. A. (2005). The effect of normalization norms in multiple attribute decision making models: a case study in gear material selection. Structural and multidisciplinary optimization, 29 (4), 312-318.

[13] Yoon, K. P., \& Hwang, C. L. (1995). Multiple attribute decision making: an introduction (Vol. 104). Sage publications.

[14] Wang, T. C., \& Lee, H. D. (2009). Developing a fuzzy TOPSIS approach based on subjective weights and objective weights. Expert Systems with Applications, 36 (5), 8980-8985. 
[15] Saaty, T. L. (1988). What is the analytic hierarchy process?. In Mathematical models for decision support (pp. 109-121). Springer Berlin Heidelberg.

[16] Zeleny, M., \& Cochrane, J. L. (1973). Multiple criteria decision making. University of South Carolina Press.

[17] Liu, S., Chan, F. T., \& Ran, W. (2016). Decision making for the selection of cloud vendor: An improved approach under group decision-making with integrated weights and objective/subjective attributes. Expert Systems with Applications, 55, 37-47.

[18] Durrett, R. (1996). Probability: theory and examples. Cambridge U Press, 39 (5), 320-353. 